\title{
Effect of Dietary Advanced Glycation end Products on Cell Cycle of Peripheral Blood Lymphocytes
}

\section{Anupriya Chhabra1, Anil Kumar Ram², Alka Bhatia ${ }^{1 *}$ and Sumit Goel ${ }^{1}$}

${ }^{1}$ Department of Experimental Medicine and Biotechnology, Postgraduate Institute of Medical Education and Research, Chandigarh, India

${ }^{2}$ Department of Immunopathology, Postgraduate Institute of Medical Education and Research, Chandigarh, India

\begin{abstract}
Advanced glycation end products (AGEs) have been linked with autoimmune diseases. AGEs present in diet are known to be absorbed intestinally. We aimed to study effects of dietary AGEs present in repeatedly heated cooking oil on cell cycle profile of lymphocytes derived from healthy volunteers. AGEs were extracted by using Aqueous-TCAChloroform method and incubated with lymphocytes for 24 hours. All extracts showed ASF (15.42-75.18) between 355-440 nm wavelength. On incubation with AGEs, an increase in S-phase fraction of treated lymphocytes $(7.67 \pm$ 3.25) was observed in 10/10 samples analysed as compared to untreated lymphocytes (1.5 \pm 1.6$)$.

The results suggest that dietary AGEs may have a role in triggering the proliferation of lymphocytes. Larger studies are required to explore role of dietary AGEs on properties of lymphocytes which may contribute to etiopathogenesis of autoimmune diseases. Such studies may provide us with a modifiable risk factor which may be altered to prevent autoimmune diseases.
\end{abstract}

Keywords: Advanced glycation end products; Fluorescence; Diet; Lymphocytes; Cell cycle

\section{Introduction}

Advanced glycation end products (AGEs) are the compounds formed due to non-enzymatic glycation of proteins, lipids or nucleic acids. Though, AGEs may form endogenously in the body as a result of normal metabolism, diet remains an important source of exogenous AGEs. Amongst the dietary AGEs, carboxymethyl lysine has been most commonly studied. Processing/cooking of foodstuffs under conditions of dry heat contributes to high AGE content of diets. Approximately $10-30 \%$ of AGEs present in the diet have been demonstrated to be absorbed intestinally. One third of absorbed AGEs are excreted via kidney and in stools. The accumulation of AGEs in body has been linked to several inflammatory and autoimmune disorders like rheumatoid arthritis, diabetes mellitus, myasthenia gravis, autoimmune uveitis etc. [1-3]. Although, indirect evidences suggest the role of dietary AGEs in etiology of inflammatory/autoimmune disorders a direct proof of principle for the same is lacking [4]. More recently, it has been established that consumption of AGE-rich diet leads to a significant rise in circulating AGE levels [5]. A reduction in dietary AGEs has also been shown to be associated with reduced oxidative stress and inflammation in patients with diabetes and renal disorders [6,7]. Most of the work on association of dietary AGEs and diseases has been done in diabetes and renal failure. In this work we have tried to study the effects of dietary AGEs on cell cycle profile of lymphocytes derived from healthy volunteers.

\section{Materials and Methods}

The AGEs were extracted from 10 samples of cooking oils subjected to repeated heating for frying different snacks by using AqueousTCA-Chloroform method. Briefly, $3 \mathrm{~mL}$ of oil sample was mixed and incubated with distilled water resulting in diffusion of water soluble AGEs in the aqueous layer. Subsequently, the proteins were precipitated out with Trichloroacetic acid (TCA, $13.5 \mathrm{M}, 1 \mathrm{~mL}$ at $4^{\circ} \mathrm{C}$ for 10 minutes). The sample was then centrifuged (Thermo electron corporation; Sorvall ${ }^{\circ}$ RC6PLUS) at $14,000 \mathrm{rpm}$ for 10 minutes at room temperature. The supernatant was carefully separated from the protein pellet and equal volume of chloroform was added (to remove the lipids) followed by centrifugation at $14,000 \mathrm{rpm}$ for 10 minutes at room temperature. The presence of AGEs in the extracts was confirmed by polyacrylamide Gel Electrophoresis and Per Iodic Acid Schiff staining which specifically stains glycoproteins and by determining AGE specific fluorescence (ASF) using fluorescence spectrophotometer (Elegant;Varian, Cary Eclipse, Excitation wavelength $355 \mathrm{~nm}$ and Emission wavelength $440 \mathrm{~nm}$ ) [8]. The lymphocytes were isolated from $10 \mathrm{~mL}$ of blood samples by Ficolle Hypaque method. They were then incubated with AGEs $(20 \mu \mathrm{L})$ extracted from cooking oil under culture conditions $\left(37^{\circ} \mathrm{C}, 5 \% \mathrm{CO} 2\right)$ for 24 hours. At the end of the incubation period, effect on cell cycle was assessed by propidium iodide staining and flow cytometry (FACS Calibur San Jose USA) using CELL QUEST software. After evaluating at least 20,000 events, the percentage of cells in synthetic phase of cell cycle were taken as S-phase fraction.

\section{Results}

All extracts showed ASF at the wavelengths mentioned above. The ASF values ranged from 15.42- 75.18 (Table 1). On incubation with AGEs, an increase in S-phase fraction (SPF) of the treated lymphocytes (Mean=7.67 \pm 3.25 ) was observed in all 10 samples analysed as compared to untreated control lymphocyte population $(1.5 \pm 1.6)$ (Figure 1). However, no relation was observed between ASF values and SPF.

\section{Discussion}

The effects of AGEs in autoimmune diseases have been attributed

*Corresponding author: Alka Bhatia, Department of Experimental Medicine and Biotechnology, Research Block B, PGIMER, Chandigarh, India, Tel: 0172 2755271; E-mail: alkabhatia@ymail.com

Received February 21, 2018; Accepted March 10, 2018; Published March 16 2018

Citation: Chhabra A, Ram AK, Bhatia A, Goel S (2018) Effect of Dietary Advanced Glycation end Products on Cell Cycle of Peripheral Blood Lymphocytes. J Blood Lymph 8: 211. doi:10.4172/2165-7831.1000211

Copyright: (c) 2018 Chhabra A, et al. This is an open-access article distributed under the terms of the Creative Commons Attribution License, which permits unrestricted use, distribution, and reproduction in any medium, provided the original author and source are credited. 
A

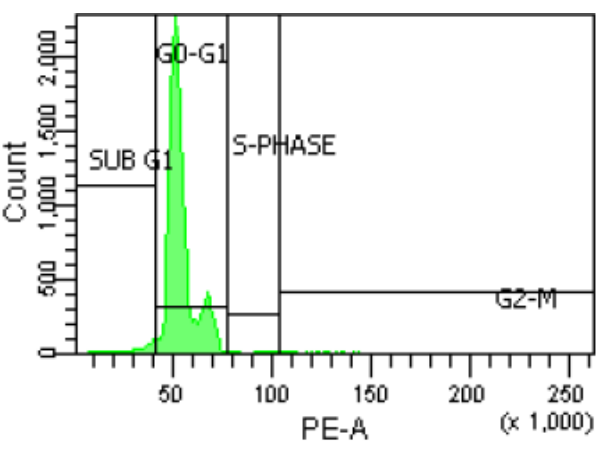

B

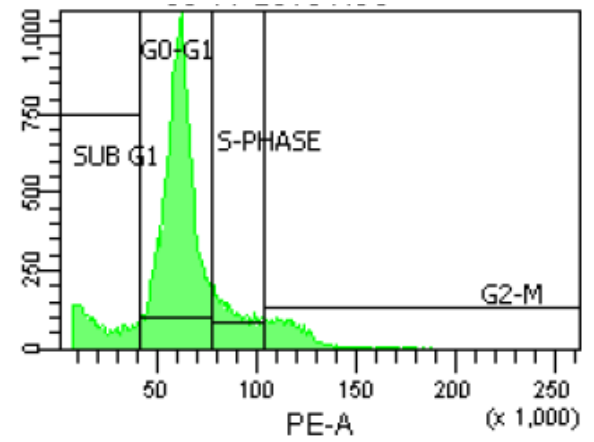

C

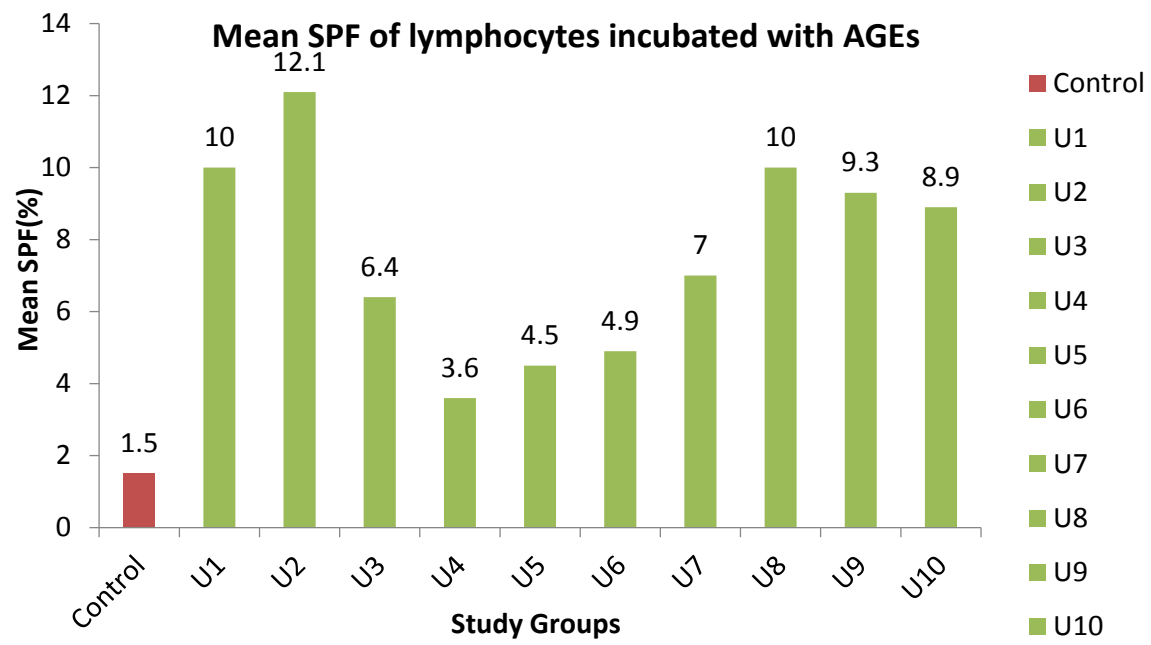

Figure 1: Representative DNA histograms of lymphocytes showing distribution of cells in different phases of cell cycle (A) Control (B) After incubation with AGEs derived from different used cooking oil samples (C) Bar diagram showing \% S-phase fraction in control and treated cells.

\begin{tabular}{|l|l|l|}
\hline SPF (control) & SPF (AGEs) & ASF \\
\hline 2.7 & $10 \%$ & 47.14 \\
\hline \multirow{5}{*}{$0.1 \%$} & 15.42 \\
\hline \multirow{3}{*}{0.3} & $3.6 \%$ & 44.14 \\
\hline & $4.5 \%$ & 75.18 \\
\hline $4.9 \%$ & 73.49 \\
\hline & $7 \%$ & 50.11 \\
\hline Mean=1.5 & $10 \%$ & 16.94 \\
\hline
\end{tabular}

Table 1: S-phase fraction in lymphocytes before and after incubation with AGEs and ASF values of cooking oil samples.

to activation of proinflammatory signalling pathways via the receptor for advanced glycation end products (RAGE). RAGE belongs to the immunoglobulin superfamily and is found on variety of cell types including macrophages, T-lymphocytes, endothelial, mesangial and smooth muscle cells. Besides AGEs, S-100 proteins, HMGB1, $\beta$-Amyloid and $\beta$-integrin also serve as ligands for RAGE. The AGERAGE axis most importantly activates NF- $\kappa B$ pathway leading to generation of proinflammatory cytokines like tumor necrosis factor- $\alpha$, interleukin-6 and C-reactive protein [3]. Therefore, AGE-RAGE axis coupled with activation of downstream signalling pathways may play an important role in propagation of inflammatory responses. Infact, past studies conducted in animal models have demonstrated that blockade of RAGE helps to reduce inflammation in the joints involved by arthritis. RAGE has also been shown to lower the threshold for activation of TLR9 which is involved in recognition of self DNA immune complexes [4].

Our results suggest that dietary AGEs have a role in triggering the proliferation of lymphocytes. In a previous study on exogenous AGEs it was shown that binding of certain type of AGEs synthesized in laboratory [Bovine serum albumin (BSA)+D-glyceraldehyde and $\mathrm{BSA}+\mathrm{D}$-glycolaldehyde] to RAGE on monocytes can induce the expression of adhesion molecules, production of cytokines like IFN- $\gamma$, TNF- $\alpha$ and lead to proliferation of T-cells [9]. As FOXp3/ROR $\gamma \mathrm{T}$ balance is believed to be altered in autoimmunity, we believe that it will be interesting to explore the effect of dietary AGEs on it [10]. Since its discovery dysregulation of IL-33/ST2 axis has been implicated in pathogenesis of different inflammatory and autoimmune diseases. The above molecules are believed to be involved in differentiation of T helper (Th) cells to Th2, Th1 or Th17 depending upon the context and cell type. Investigating the influence of dietary AGEs on the above therefore appears to be a fascinating aspect in futuristic studies $[11,12]$.

As dietary AGEs can be highly heterogeneous it remains to be determined which type of AGEs in diet are responsible for modulation of our immune response and whether such modulations can lead to autoimmune diseases. Further, which genetic, epigenetic and molecular events are exactly affected and what implications it may have in autoimmunity remains to be sorted out. Also, as AGEs constitute an 
Citation: Chhabra A, Ram AK, Bhatia A, Goel S (2018) Effect of Dietary Advanced Glycation end Products on Cell Cycle of Peripheral Blood Lymphocytes. J Blood Lymph 8: 211. doi:10.4172/2165-7831.1000211

Page 3 of 3

integral part of modern diets which include many heat processed food items, the threshold level beyond which they can be hazardous needs to be determined. Many studies have also shown the protective effect of substances like lime juice, vinegar etc. in diet. Marinating the foodstuffs with the later has been found to decrease the AGE content of foods.

\section{Conclusion}

This preliminary report suggests that dietary AGEs may have important effect on lymphocyte cell cycle and hence may serve as a basis for conducting larger scale studies to explore the role of dietary AGEs in etiology and promotion of autoimmune/inflammatory diseases. Such studies may be important as this may provide us with a modifiable factor which may be altered to curb the growing menace of autoimmune diseases across the globe.

\section{References}

1. Abate G, Delbarba A, Marziano M, Memo M, Uberti D (2015) Advanced Glycation End Products (AGEs) in Food: Focusing on Mediterranean Pasta. J Nutr Food Sci 5: 440

2. Uribarri J, Woodruff S, Goodman S, Cai W, Chen X, et al. (2010) Advanced Glycation End Products in Foods and a Practical Guide to Their Reduction in the Diet. J Am Diet Assoc 110: 911-916.

3. Ott C, Jacobs K, Haucke E, Santos AN, Grune T, et al. (2014) Role of advanced glycation end products in cellular signaling. Redox Biol 2: 411-429.

4. Sirois CM, Jin T, Miller AL, Bertheloot D, Nakamura H, et al. (2013) RAGE is an nucleic acid receptor that promotes inflammatory responses to DNA. J Exp Med 210: 2447-2463.

5. Koschinsky T, He CJ, Mitsuhashi T, Bucala R, Liu C, et al. (1997) Orally absorbed reactive glycation products (glycotoxins): an environmental risk factor in diabetic nephropathy. Proc Natl Acad Sci 94: 6474-6479.

6. Vlassara H, Cai W, Crandall J, Goldberg T, Oberstein R, et al. (2002) Inflammatory mediators are induced by dietary glycotoxins, a major risk factor for diabetic angiopathy. Proc Natl Acad Sci 99: 15596-15601.

7. Uribarri J, Peppa M, Cai W, Goldberg T, Lu M, et al. (2003) Restriction of dietary glycotoxins reduces excessive advanced glycation end products in renal failure patients. J Am Soc Nephrol 14: 728-731.

8. Maza MP, Garrido F, Escalante N, Leiva L, Barrera G, et al. (2012) Fluorescent advanced glycation end-products (ages) detected by spectro-photofluorimetry as a screening tool to detect diabetic microvascular complications. J Diabetes Mellitus 2: 221-226.

9. Ohashi K, Takahashi HK, Mori S, Liu K, Wake H, et al. (2010) Advanced glycation end products enhance monocyte activation during human mixed lymphocyte reaction. Clinical Immunology 134: 345-353.

10. Ichiyama K, Yoshida H, Wakabayashi Y, Chinen T, Saeki K, et al. (2008) Foxp3 Inhibits RORyt-mediated IL-17A mRNA Transcription through Direct Interaction with RORyt. The Journal of Biological Chemistry 283: 17003-17008.

11. Ciccone MM, Cortese F, Gesualdo M, Riccardi R, Nunzio DD, et al. (2013) A Novel Cardiac Bio-Marker: ST2: A Review. Molecules 18: 15314-15328.

12. Pei C, Barbour M, Fairlie-Clarke KJ, Allan D, Mu R, et al. (2014) Emerging role of interleukin-33 in autoimmune diseases. Immunology 141: 9-17. 